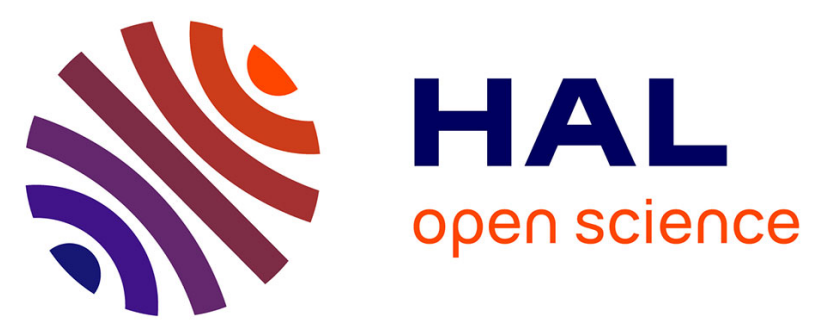

\title{
Leaderless Consensus-based Formation Control of Multiple Nonholonomic Mobile Robots with Interconnecting Delays
}

Emmanuel Nuno, Tomatiuh Hernandez, Mohamed Maghenem, Antonio Loria, Elena Panteley

\section{To cite this version:}

Emmanuel Nuno, Tomatiuh Hernandez, Mohamed Maghenem, Antonio Loria, Elena Panteley. Leaderless Consensus-based Formation Control of Multiple Nonholonomic Mobile Robots with Interconnecting Delays. ACC 2019 - American Control Conference, Jul 2019, Philadelphia, PA, United States. pp.4659-4664, 10.23919/ACC.2019.8814391 . hal-02367891

\section{HAL Id: hal-02367891 \\ https://hal.science/hal-02367891}

Submitted on 5 Mar 2020

HAL is a multi-disciplinary open access archive for the deposit and dissemination of scientific research documents, whether they are published or not. The documents may come from teaching and research institutions in France or abroad, or from public or private research centers.
L'archive ouverte pluridisciplinaire HAL, est destinée au dépôt et à la diffusion de documents scientifiques de niveau recherche, publiés ou non, émanant des établissements d'enseignement et de recherche français ou étrangers, des laboratoires publics ou privés. 


\title{
Leaderless Consensus-based Formation Control of Multiple Nonholonomic Mobile Robots with Interconnecting Delays
}

\author{
Emmanuel Nuño ${ }^{1}$, Tonatiuh Henández ${ }^{1}$, Mohamed Maghenem ${ }^{2}$, Antonio Loría ${ }^{3}$, Elena Panteley ${ }^{3,4}$
}

\begin{abstract}
The control objective in the leaderless consensusbased formation control is to ensure that the Cartesian positions of the nonholonomic mobile robots converge to a given position in a formation pattern and the barycentre of such formation is agreed, in a decentralized manner, by all the robots, while their orientations converge to a common value. The main problem behind this stabilization objective is that for robots that exhibit nonholonomic restrictions, due to the Brocket's condition, the controller has to be designed such that it is discontinuous or it is non-autonomous (time-varying). In this work we propose a simple Proportional plus damping $(\mathbf{P}+\mathbf{d})$ smooth controller that solves the aforementioned objective and we provide a sufficient condition on the damping gain to ensure robustness with respect to variable time-delays in the interconnection. Simulations are provided to show the effectiveness of our control proposal.
\end{abstract}

\section{INTRODUCTION}

The control objective in the consensus of multiple dynamical agents is to ensure that the states of all agents agree on a common value by sharing (part of) their state with their corresponding neighbors [1], [2], [3], [4]. There are mainly two different consensus problems: the leader-follower, in which the agreement value is given as a desired value to a set of (follower) agents in the network; and the leaderless, in which such common value if found among the agents [5], [6], [7]. In this work we deal with the consensus-based formation control of a class of nonholonomic mobile robots, where the control objective is to drive the Cartesian positions of all the robots to a given position in a formation pattern and the barycentre of such formation is agreed upon by all the robots in a decentralized fashion. Furthermore, the robot orientations converge to a common consensus value.

Consensus of nonholonomic mobile robots has been studied, for instance, in [8] where a decentralized feedback control that drives a system of multiple nonholonomic unicycles to a rendezvous point in terms of both position and orientation is proposed, the control law is discontinuous and time-invariant. In [9] necessary and sufficient conditions for the feasibility of a class of position formations are laid. In [10] the position/orientation formation control problem for multiple nonholonomic agents using a time-varying controller that leads the agents to a given formation using only their orientation is proposed. In [11] a distributed consensus control law for a network of nonholonomic agents in the

\footnotetext{
${ }^{1}$ Department of Computer Science, CUCEI. University of Guadalajara. Guadalajara, Mexico. E-mail: emmanuel.nuno@cucei.udg.mx

${ }^{2}$ University of California at Santa Cruz. California, USA.

${ }^{3}$ L2S-CentraleSupelec, CNRS, 3 Rue Joliot Curie, Gif-sur-Yvette, France.

${ }^{4}$ ITMO University, Kronverkskiy av. 49, Saint Petersburg, 197101, Russia.
}

presence of bounded disturbances with unknown dynamics in all inputs channels is presented. In [12] a smooth distributed formation control law using a consensus-based approach to drive a group of agents to a desired geometric pattern is proposed. The latter result is extended in [13] by introducing a Proportional plus damping $(\mathrm{P}+\mathrm{d})$ controller for the velocity dynamics. In [14] the consensus-based formation problem is solved using a time-varying controller and the solution is enabled with collision avoidance capabilities. However, in all of these references it is assumed that the communications are reliable and a simplified kinematics model of the nonholonomic mobile robots is used.

In contrast to the latter, in [15], [16] and [17] a more realistic torque-controlled second-order dynamics model is used, but it is assumed that communications are reliable (without delays). The consensus problem is also addressed in [18] via a cooperative control law that is robust to constant communication delays. However, in the latter reference, a first-order kinematics model is used.

In this article we solve the consensus-based formation control problem for a class of nonholonomic mobile robots interconnected through unreliable communication channels that exhibit variable time-delays. In addition, we use a second-order torque-controlled model for the robots. Our controller is simple to implement, as it consists in a smooth controller of the Proportional plus damping $(\mathrm{P}+\mathrm{d})$ type. To the best of the authors' knowledge this is the first work on consensus of mobile robots, in which the full secondorder dynamic model is used and variable time-delays in the communications are taken into account.

Notation. $\mathbb{R}:=[-\infty, \infty], \mathbb{R}_{>0}:=(0, \infty], \mathbb{R}_{\geq 0}:=[0, \infty]$. $\|\mathbf{x}\|$ stands for the standard Euclidean norm of vector $\mathbf{x}$. $\mathbf{I}_{n}$ represents the identity matrix of size $n \times n . \mathbf{1}_{k}$ and $\mathbf{0}_{k}$ represent column vectors of size $k$ with all entries equal to one and to zero, respectively. $\otimes$ represents the standard Kronecker product. For any function $\mathbf{f}: \mathbb{R}_{>0} \rightarrow \mathbb{R}^{n}$, the $\mathcal{L}_{\infty^{-}}$ norm is defined as $\|\mathbf{f}\|_{\infty}:=\sup _{t>0}|\mathbf{f}(t)|, \mathcal{L}_{2}$-norm as $\|\mathbf{f}\|_{2}:=$ $\left(\int_{0}^{\infty}|\mathbf{f}(t)|^{2} d t\right)^{1 / 2}$. The $\mathcal{L}_{\infty}$ and $\mathcal{L}_{2}$ spaces are defined as the sets $\left\{\mathbf{f}: \mathbb{R}_{\geq 0} \rightarrow \mathbb{R}^{n} \mid\|\mathbf{f}\|_{\infty}<\infty\right\}$ and $\left\{\mathbf{f}: \mathbb{R}_{>0} \rightarrow\right.$ $\left.\mathbb{R}^{n} \mid\|\mathbf{f}\|_{2}<\infty\right\}$, respectively. The set $\bar{N}$ is defined as $\bar{N}:=$ $\{1, \ldots, N\}$, where $N$ is a positive natural number.

\section{Problem Setting}

We consider a swarm of $N$ nonholonomic vehicles modeled as differential drive robots that move in the Cartesian $x y$-plane with three degrees of freedom, two translations and one rotation. Let us define $\mathbf{z}_{i}:=\left[x_{i}, y_{i}\right]^{\top} \in \mathbb{R}^{2}$ 
and $\theta_{i} \in \mathbb{R}$ as the translation and rotation coordinates, respectively, of the $i$ th-robot. Let us also define $\boldsymbol{\delta}_{i} \in \mathbb{R}^{2}$ as the relative desired translation of the $i$ th-robot with regards to the barycentre of a given formation pattern. Thus, the corresponding position of the $i$ th-robot translated to the barycentre of the desired formation is $\overline{\mathbf{z}}_{i}:=\mathbf{z}_{i}-\boldsymbol{\delta}_{i}$.

The interconnection of the mobile robots is modelled using the Laplacian matrix $\mathbf{L}:=\left[\ell_{i j}\right] \in \mathbb{R}^{N \times N}$, whose elements are defined as

$$
\ell_{i j}=\left\{\begin{array}{cc}
\sum_{k \in \mathcal{N}_{i}} a_{i k} & i=k \\
-a_{i k} & i \neq k
\end{array}\right.
$$

where $i \in \bar{N}$ and $\mathcal{N}_{i}$ is the set of transmitting information to the $i$ th robot, $a_{i k}>0$ if $k \in \mathcal{N}_{i}$ and $a_{i k}=0$ otherwise.

Similar to passivity-based (energy-shaping) synchronization [19], [20], [21], the following assumption is used in this paper:

A1. The interconnection graph is undirected, static and connected.

Remark 1: Note that, by construction, $\mathbf{L}$ has a zero row sum, i.e., $\mathbf{L} \mathbf{1}_{N}=\mathbf{0}_{N}$. Moreover, Assumption A1, ensures that $\mathbf{L}$ is symmetric, has a single zero-eigenvalue and the rest of the spectrum of $\mathbf{L}$ is strictly positive. Thus, $\operatorname{rank}(\mathbf{L})=$ $N-1$. Therefore, the only vector living in the kernel of $\mathbf{L}$ is $\operatorname{span}\left(\mathbf{1}_{N}\right)$.

The information exchange between the vehicles is subjected to time-delays that satisfy the following assumption.

A2. The communication from the $j$ th to the $i$ th robot is subject to a variable time-delay denoted $T_{j i}(t)$ which is bounded by a known upper-bound ${ }^{*} T_{j i} \geq 0$. Further, $T_{j i}(t)$ has, up to the fourth, bounded time-derivatives.

The control objective in this paper is the following:

(LFP) Leaderless Formation Problem. Consider a swarm of $N$ nonholonomic vehicles modeled as differential drive robots. Under Assumptions A1 and A2, design a decentralized controller such that, given a desired formation pattern, all the robots (globally and asymptotically) agree on their relative Cartesian positions and orientations, i.e., for all $\mathbf{z}_{i} \in \mathbb{R}^{2}$ and $\theta_{i} \in \mathbb{R}$

$$
\lim _{t \rightarrow \infty}\left[\begin{array}{c}
\overline{\mathbf{z}}_{i}(t) \\
\theta_{i}(t)
\end{array}\right]=\left[\begin{array}{c}
\overline{\mathbf{z}}_{c} \\
\theta_{c}
\end{array}\right],
$$

where $\overline{\mathbf{z}}_{c} \in \mathbb{R}^{2}$ is the Cartesian consensus position of the barycentre of the formation and $\theta_{c} \in \mathbb{R}$ is the consensus orientation of the robots.

\section{DYNAMIC MODEL}

We assume that for each robot, the geometrical center and the center of mass are located at the same point $\mathrm{Q}:=$ $\left[x_{i}, y_{i}\right]^{\top}$. Then, the corresponding dynamics of the $i$ th-robot is given by

$$
\begin{aligned}
\dot{\mathbf{z}}_{i} & =\left[\begin{array}{c}
\cos \left(\theta_{i}\right) \\
\sin \left(\theta_{i}\right)
\end{array}\right] v_{i}, \\
\dot{\theta}_{i} & =\omega_{i}, \\
{\left[\begin{array}{c}
\dot{v}_{i} \\
\dot{\omega}_{i}
\end{array}\right] } & =\left[\begin{array}{cc}
\frac{1}{m_{i}} & 0 \\
0 & \frac{1}{I_{i}}
\end{array}\right] \mathbf{B}_{i} \boldsymbol{\tau}_{i}
\end{aligned}
$$

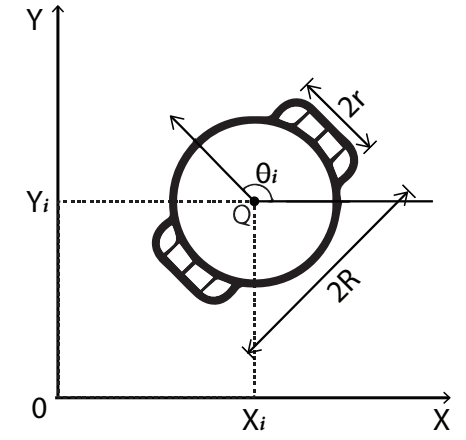

Fig. 1. Schematics of a differential wheeled mobile robot.

where $v_{i}$ and $\omega_{i}$ are the linear and the angular velocities of the center of mass, respectively; $m_{i}$ is the mass; $I_{i}$ is the moment of inertia; $R$ is the distance between point $\mathbf{Q}$ and the wheels; and $r$ the radius of the latter. $\tau_{i}$ is the control input torque of the left and right wheels, i.e., $\boldsymbol{\tau}_{i}=\left[\tau_{i l}, \tau_{i r}\right]^{\top}$, and $\mathbf{B}_{i}=\frac{1}{r}\left[\begin{array}{cc}1 & 1 \\ R & -R\end{array}\right]$-see Fig. 1 .

A first step in the controller design is to propose the following inner control-loop

$$
\boldsymbol{\tau}_{i}=\mathbf{B}_{i}^{-1} \mathbf{u}_{i}=\frac{r}{2}\left[\begin{array}{cc}
1 & \frac{1}{R} \\
1 & -\frac{1}{R}
\end{array}\right]\left[\begin{array}{l}
u_{v i} \\
u_{\omega i}
\end{array}\right],
$$

where the extra input term $\mathbf{u}_{i} \in \mathbb{R}^{2}$ will be defined in the next section. The closed-loop (2) and (3) yields

$$
\begin{aligned}
\dot{\mathbf{z}}_{i} & =\boldsymbol{\varphi}\left(\theta_{i}\right) v_{i}, \\
\dot{\theta}_{i} & =\omega_{i}, \\
\dot{v}_{i} & =\frac{1}{m_{i}} u_{v i} \\
\dot{\omega}_{i} & =\frac{1}{I_{i}} u_{\omega i},
\end{aligned}
$$

where

$$
\varphi\left(\theta_{i}\right)=\left[\begin{array}{c}
\cos \left(\theta_{i}\right) \\
\sin \left(\theta_{i}\right)
\end{array}\right]
$$

\section{Distributed Consensus-BAsed Formation CONTROL}

The extra input term $\mathbf{u}_{i}$ of the decentralized controller that solves the (LFP) problem is composed of two smooth $\mathrm{P}+\mathrm{d}$ elements that drive the linear and angular accelerations, respectively. The first element is

$$
u_{v i}=-p_{v i} \boldsymbol{\varphi}^{\top}\left(\theta_{i}\right) \mathbf{e}_{i}-d_{v i} v_{i}
$$

where $p_{v i}, d_{v i}>0$ are the proportional and the damping gains, respectively; and $\mathbf{e}_{i}$ is the Cartesian position error of the $i$ th-robot, with regards to its neighbors, defined as

$$
\mathbf{e}_{i}:=\sum_{j \in \mathcal{N}_{i}} a_{i j}\left(\overline{\mathbf{z}}_{i}-\overline{\mathbf{z}}_{j}\left(t-T_{j i}(t)\right)\right) .
$$

The second element is

$$
u_{\omega i}=-p_{\omega i} e_{\theta i}-d_{\omega i} \omega_{i}+\alpha_{i}\left(t, \theta_{i}, \mathbf{e}_{i}\right),
$$


where $p_{\omega i}, d_{\omega i}>0$ are the proportional and the damping gains, respectively; $e_{\theta i}$ is the orientation error of the $i$ throbot, with regards to its neighbors, given by

$$
e_{\theta i}:=\sum_{j \in \mathcal{N}_{i}} a_{i j}\left(\theta_{i}-\theta_{j}\left(t-T_{j i}(t)\right)\right),
$$

and $\alpha_{i} \in \mathbb{R}$ is a smooth time-varying function that is included to satisfy Brockett's condition and that will be designed later.

The complete closed-loop system given by (2), (3), (6) and (8) is

$$
\begin{aligned}
& \Sigma_{v}\left\{\begin{array}{l}
\dot{\mathbf{z}}_{i}=\boldsymbol{\varphi}\left(\theta_{i}\right) v_{i} \\
\dot{v}_{i}=-\frac{1}{m_{i}}\left[p_{v i} \boldsymbol{\varphi}^{\top}\left(\theta_{i}\right) \mathbf{e}_{i}+d_{v i} v_{i}\right] \quad \forall i \in \bar{N},
\end{array}\right. \\
& \Sigma_{\omega}\left\{\begin{array}{l}
\dot{\theta}_{i}=\omega_{i} \\
\dot{\omega}_{i}=-\frac{1}{I_{i}}\left[p_{\omega i} e_{\theta i}+d_{\omega i} \omega_{i}-\alpha_{i}\left(t, \theta_{i}, \mathbf{e}_{i}\right)\right]
\end{array} \quad \forall i \in \bar{N} .\right.
\end{aligned}
$$

Next, we establish some important properties for $\Sigma_{\omega}$, regarded as a linear autonomous system with variable timedelays and driven by the "input" $\alpha_{i}\left(t, \theta_{i}, \mathbf{e}_{i}\right)$.

Proposition 1: Suppose that Assumptions A1 and A2 hold. Set the damping gain $d_{\omega i}$ such that

$$
2 d_{\omega i}>p_{\omega i} \sum_{j \in \mathcal{N}_{i}} a_{i j}\left(\beta_{i}+\frac{* T_{i j}^{2}}{\beta_{j}}\right), \quad \forall i \in \bar{N},
$$

for some $\left\{\beta_{1}, \ldots, \beta_{N}\right\}>0$. In this scenario:

1) if $\alpha_{i}\left(t, \theta_{i}, \mathbf{e}_{i}\right)=0$ then, for $\Sigma_{\omega}$ in (10), there exists $\theta_{c} \in \mathbb{R}$ such that $\left(\omega_{i}, \theta_{i}\right)=\left(0, \theta_{c}\right)$ is Globally Asymptotically Stable (GAS);

2) if $\alpha_{i} \in \mathcal{L}_{\infty}$ then $\dot{\omega}_{i}, \omega_{i} \in \mathcal{L}_{\infty}$;

$3)$ if $\alpha_{i} \in \mathcal{L}_{\infty}$ and $\lim _{t \rightarrow \infty} \alpha_{i}\left(t, \theta_{i}(t), \mathbf{e}_{i}(t)\right)=0$ then $\lim _{t \rightarrow \infty} \omega_{i}(t)=0$ and $\lim _{t \rightarrow \infty}^{t \rightarrow \infty} \theta_{i}(t)=\theta_{c}$.

Proof. To establish item 1 ) ${ }^{t \rightarrow \infty}$ of the proposition we set $\alpha_{i}=$ 0 in $\Sigma_{\omega}$. Consider the Lyapunov-Krasovskii functional

$$
\begin{aligned}
\mathcal{V}:=\sum_{i=1}^{N}[ & \frac{I_{i}}{2 p_{\omega i}} \omega_{i}^{2}+\frac{1}{4} \sum_{j \in \mathcal{N}_{i}} a_{i j}\left(\theta_{i}-\theta_{j}\right)^{2} \\
& \left.+c_{i} \sum_{j \in \mathcal{N}_{i}} a_{i j} \int_{-* T_{j i}}^{0} \int_{t+\sigma}^{t} \omega_{j}^{2}(\eta) d \eta d \sigma\right],
\end{aligned}
$$

where $c_{i}$ is a positive number. $\dot{\mathcal{V}}$ is given by

$$
\begin{aligned}
\dot{\mathcal{V}}=\sum_{i=1}^{N} & {\left[\frac{I_{i}}{p_{\omega i}} \omega_{i} \dot{\omega}_{i}+\frac{1}{2} \sum_{j \in \mathcal{N}_{i}} a_{i j}\left(\dot{\theta}_{i}-\dot{\theta}_{j}\right)\left(\theta_{i}-\theta_{j}\right)\right.} \\
& \left.+c_{i} \sum_{j \in \mathcal{N}_{i}} a_{i j}\left({ }^{*} T_{j i} \omega_{j}^{2}-\int_{t-{ }^{*} T_{j i}}^{t} \omega_{j}^{2}(\sigma) d \sigma\right)\right] .
\end{aligned}
$$

Evaluating $\dot{\mathcal{V}}$ along $\Sigma_{\omega}$, in (10), yields

$$
\begin{aligned}
\dot{\mathcal{V}}=-\sum_{i=1}^{N} & {\left[\frac{d_{\omega i}}{p_{\omega i}} \omega_{i}^{2}+\sum_{j \in \mathcal{N}_{i}} a_{i j} \omega_{i} \int_{t-T_{j i}(t)}^{t} \omega_{j}(\sigma) d \sigma\right.} \\
& \left.-c_{i} \sum_{j \in \mathcal{N}_{i}} a_{i j}\left({ }^{*} T_{j i} \omega_{j}^{2}-\int_{t-* T_{j i}}^{t} \omega_{j}^{2}(\sigma) d \sigma\right)\right],
\end{aligned}
$$

where, to obtain this equation, we have used the fact that

$$
\theta_{j}-\theta_{j}\left(t-T_{j i}(t)\right)=\int_{t-T_{j i}(t)}^{t} \dot{\theta}_{j}(\sigma) d \sigma=\int_{t-T_{j i}(t)}^{t} \omega_{j}(\sigma) d \sigma
$$

and we have also invoked $\mathbf{A} \mathbf{1}$ and the properties of the Laplacian matrix to prove that

$$
\sum_{i=1}^{N} \sum_{j \in \mathcal{N}_{i}} a_{i j}\left(\dot{\theta}_{i}+\dot{\theta}_{j}\right)\left(\theta_{i}-\theta_{j}\right)=0
$$

It also holds that, for any $\beta_{i}>0$,

$$
\begin{aligned}
-\omega_{i} \int_{t-T_{j i}(t)}^{t} \omega_{j}(\sigma) d \sigma & \leq \frac{\beta_{i}}{2} \omega_{i}^{2}+\frac{1}{2 \beta_{i}}\left|\int_{t-T_{j i}(t)}^{t} \omega_{j}(\sigma) d \sigma\right|^{2} \\
& \leq \frac{\beta_{i}}{2} \omega_{i}^{2}+\frac{* T_{j i}}{2 \beta_{i}} \int_{t-* T_{j i}}^{t} \omega_{j}^{2}(\sigma) d \sigma .
\end{aligned}
$$

Setting $c_{i}=\frac{{ }^{*} T_{j i}}{2 \beta_{i}}$ returns

$$
\dot{\mathcal{V}} \leq-\sum_{i=1}^{N}\left[\frac{d_{\omega i}}{p_{\omega i}} \omega_{i}^{2}-\sum_{j \in \mathcal{N}_{i}} a_{i j}\left(\frac{\beta_{i}}{2} \omega_{i}^{2}+\frac{* T_{j i}^{2}}{2 \beta_{i}} \omega_{j}^{2}\right)\right]
$$

and using $l_{i i}:=\sum_{j \in \mathcal{N}_{i}} a_{i j}$, where $l_{i i}$ is the diagonal element of the Laplacian, we obtain

$$
\dot{\mathcal{V}} \leq-\sum_{i=1}^{N}\left[\left(\frac{d_{\omega i}}{p_{\omega i}}-\frac{\beta_{i}}{2} l_{i i}\right) \omega_{i}^{2}-\sum_{j \in \mathcal{N}_{i}} a_{i j} \frac{{ }^{*} T_{j i}^{2}}{2 \beta_{i}} \omega_{j}^{2}\right]
$$

As in [19], defining $\mathbf{W}:=\operatorname{col}\left(\omega_{1}^{2}, \ldots, \omega_{N}^{2}\right)$ and

$$
\Psi=\left[\begin{array}{cccc}
\frac{d_{\omega 1}}{p_{\omega 1}}-\frac{\beta_{1}}{2} l_{11} & -\frac{{ }^{*} T_{21}^{2}}{2 \beta_{1}} a_{12} & \ldots & -\frac{{ }^{*} T_{N 1}^{2}}{2 \beta_{1}} a_{1 N} \\
-\frac{{ }^{*} T_{12}^{2}}{2 \beta_{2}} a_{21} & \frac{d_{\omega 2}}{p_{\omega 2}}-\frac{\beta_{2}}{2} l_{22} & \ldots & -\frac{{ }^{*} T_{N 2}^{2}}{2 \beta_{2}} a_{2 N} \\
\vdots & \vdots & \ddots & \vdots \\
-\frac{{ }^{*} T_{1 N}^{2}}{2 \beta_{N}} a_{N 1} & -\frac{{ }^{*} T_{2 N}^{2}}{2 \beta_{N}} a_{N 2} & \ldots & \frac{d_{\omega N}}{p_{\omega N}}-\frac{\beta_{N}}{2} l_{N N}
\end{array}\right]
$$

we can write $\dot{\mathcal{V}} \leq-\mathbf{1}_{N}^{\top} \mathbf{\Psi} \mathbf{W}$ or, equivalently,

$$
\dot{\mathcal{V}} \leq-\sum_{i=1}^{N}\left(\frac{d_{\omega i}}{p_{\omega i}}-\sum_{j \in \mathcal{N}_{i}} a_{i j}\left(\frac{\beta_{i}}{2}+\frac{{ }^{*} T_{i j}^{2}}{2 \beta_{j}}\right)\right) \omega_{i}^{2}
$$

Then, setting $d_{\omega i}$ such that (11) holds, it follows that there exists $\lambda_{i}>0$ such that $\dot{\mathcal{V}} \leq-\sum_{i=1}^{N} \lambda_{i} \omega_{i}^{2}$. Thus $\omega_{i} \in \mathcal{L}_{2}$. Further, since $\mathcal{V}$ is positive definite and radially unbounded with regards to $\omega_{i}$ and $\theta_{i}-\theta_{j}$, then these signals are bounded. Which, in turn, imply that $\dot{\omega}_{i} \in \mathcal{L}_{\infty}$. Invoking Barbalat's Lemma it is established that $\lim _{t \rightarrow \infty} \omega_{i}(t)=0$. Obviously, from $\Sigma_{\omega}$ in (10), if we can prove that $\lim _{t \rightarrow \infty} \dot{\omega}_{i}(t)=0$ then $\lim _{t \rightarrow \infty} e_{\theta i}(t)=0$. This last, in turn, implies that

$$
\lim _{t \rightarrow \infty} \sum_{j \in \mathcal{N}_{i}} a_{i j}\left(\theta_{i}(t)-\theta_{j}(t)+\int_{t-T_{j i}(t)}^{t} \omega_{j}(\sigma) d \sigma\right)=0 .
$$


Since $\omega_{i}$ converges for all $i \in\{1, \ldots, N\}$ and $T_{j i}(t)$ is globally bounded, then

$$
\lim _{t \rightarrow \infty} \sum_{j \in \mathcal{N}_{i}} a_{i j}\left(\theta_{i}(t)-\theta_{j}(t)\right)=0 .
$$

Defining $\boldsymbol{\theta}:=\left[\theta_{1}, \ldots, \theta_{N}\right]^{\top} \in \mathbb{R}^{N}$, then we have that $\lim _{t \rightarrow \infty} \mathbf{L} \boldsymbol{\theta}(t)=\mathbf{0}$ which implies that $\lim _{t \rightarrow \infty} \theta_{i}(t)=\theta_{c}$.

Now, since

$$
\lim _{t \rightarrow \infty} \int_{0}^{t} \dot{\omega}_{i}(\sigma) d \sigma=\lim _{t \rightarrow \infty} \omega_{i}(t)-\omega_{i}(0)=\omega_{i}(0),
$$

then such limit exists. Moreover,

$\ddot{\omega}_{i}=-\frac{1}{I_{i}}\left[p_{\omega i} \sum_{j \in \mathcal{N}_{i}} a_{i j}\left(\omega_{i}-\left(1-\dot{T}_{j i}\right) \omega_{j}\left(t-T_{j i}(t)\right)\right)+d_{\omega i} \dot{\omega}_{i}\right]$.

which, in view of Assumption $\mathbf{A 2}$ and the fact that $\dot{\omega}_{i}, \omega_{i} \in$ $\mathcal{L}_{\infty}$ imply that $\dot{\omega}_{i}$ is uniformly continuous. Thus, by Barbalat's Lemma $\omega_{i}$ asymptotically converges to zero. This completes the proof of item 1).

Next, we establish item 2). To that end, we first recall that without input the equilibrium $\left(\omega_{i}, \theta_{i}\right)=\left(0, \theta_{c}\right)$ is GAS. Further $\Sigma_{\omega}$ is a marginally stable linear time-varying system with uniformly bounded time-delays. Hence, by Proposition 3 in [22] if $\alpha_{i}\left(t, \theta_{i}, \mathbf{e}_{i}\right)$ is bounded then $\dot{\omega}_{i}, \omega_{i} \in \mathcal{L}_{\infty}$.

Item 3 ) is established also by Proposition 3 in [22]. This finishes the proof of the proposition.

In what follows we establish the fact that $\left(v_{i}, \varphi^{\top}\left(\theta_{i}\right) \mathbf{e}_{i}\right)=$ $(0,0)$ is GAS, for system $\Sigma_{v}$ in (10).

Proposition 2: Suppose that Assumptions A1 and A2 hold. Set the damping gain $d_{v i}$ such that

$$
2 d_{v i}>p_{v i} \sum_{j \in \mathcal{N}_{i}} a_{i j}\left(\beta_{i}+\frac{{ }^{*} T_{i j}^{2}}{\beta_{j}}\right), \quad \forall i \in \bar{N},
$$

for some $\left\{\beta_{1}, \ldots, \beta_{N}\right\}>0$. Then $v_{i} \in \mathcal{L}_{\infty} \cap \mathcal{L}_{2}$, $\left\|\overline{\mathbf{z}}_{i}-\overline{\mathbf{z}}_{j}\right\| \in \mathcal{L}_{\infty}$ and $\lim _{t \rightarrow \infty} v_{i}(t)=0$. If, additionally, $\omega_{i} \in$ $\mathcal{L}_{\infty}$ then $\lim _{t \rightarrow \infty} \varphi^{\top}\left(\theta_{i}(t)\right) \mathbf{e}_{i}(t)=0$.

Proof. Consider the Lyapunov-Krasovskii functional

$$
\begin{aligned}
\mathcal{W}:=\sum_{i=1}^{N} & {\left[\frac{m_{i}}{2 p_{v i}} v_{i}^{2}+\frac{1}{4} \sum_{j \in \mathcal{N}_{i}} a_{i j}\left\|\overline{\mathbf{z}}_{i}-\overline{\mathbf{z}}_{j}\right\|^{2}\right.} \\
& \left.+c_{i} \sum_{j \in \mathcal{N}_{i}} a_{i j} \int_{-{ }^{*} T_{j i}}^{0} \int_{t+\sigma}^{t} v_{j}^{2}(\eta) d \eta d \sigma\right] .
\end{aligned}
$$

whose total derivative yields

$$
\begin{aligned}
\dot{\mathcal{W}}=\sum_{i=1}^{N} & {\left[\frac{m_{i}}{p_{v i}} v_{i} \dot{v}_{i}+\frac{1}{2} \sum_{j \in \mathcal{N}_{i}} a_{i j}\left(\dot{\mathbf{z}}_{i}-\dot{\mathbf{z}}_{j}\right)^{\top}\left(\overline{\mathbf{z}}_{i}-\overline{\mathbf{z}}_{j}\right)\right.} \\
& \left.+c_{i} \sum_{j \in \mathcal{N}_{i}} a_{i j}\left({ }^{*} T_{j i} v_{j}^{2}-\int_{t-* T_{j i}}^{t} v_{j}^{2}(\sigma) d \sigma\right)\right] .
\end{aligned}
$$

Since $\varphi^{\top}\left(\theta_{i}\right) \boldsymbol{\varphi}\left(\theta_{i}\right)=1$ then $\left\|\dot{\mathbf{z}}_{i}\right\|^{2}=v_{i}^{2}$. This last, setting $c_{i}=\frac{{ }^{*} T_{j i}}{2 \beta_{i}}$ and using the same arguments as in the proof of item 1) in Proposition 1, we get

$$
\dot{\mathcal{W}} \leq-\sum_{i=1}^{N}\left(\frac{d_{v i}}{p_{v i}}-\sum_{j \in \mathcal{N}_{i}} a_{i j}\left(\frac{\beta_{i}}{2}+\frac{{ }^{*} T_{i j}^{2}}{2 \beta_{j}}\right)\right) v_{i}^{2} .
$$

Therefore, for all $i \in \bar{N}$, setting $d_{v i}$ satisfying (12) ensures that $v_{i} \in \mathcal{L}_{\infty} \cap \mathcal{L}_{2}$ and $\left\|\overline{\mathbf{z}}_{i}-\overline{\mathbf{z}}_{j}\right\| \in \mathcal{L}_{\infty}$. This implies that $\mathbf{e}_{i} \in \mathcal{L}_{\infty}$ and, since $\varphi\left(\theta_{i}\right)$ is bounded, $\dot{v}_{i} \in \mathcal{L}_{\infty}$. Therefore, by Barbalat's Lemma, $\lim _{t \rightarrow \infty} v_{i}(t)=0$. This completes the first part of the proof.

Since

$$
\lim _{t \rightarrow \infty} \int_{0}^{t} \dot{v}_{i}(\sigma) d \sigma=\lim _{t \rightarrow \infty} v_{i}(t)-v_{i}(0)=v_{i}(0),
$$

we only need to prove that $\dot{v}_{i}$ is uniformly continuous to establish convergence of $\varphi^{\top}\left(\theta_{i}\right) \mathbf{e}_{i}$ to zero. First note that

$$
\ddot{v}_{i}=-\frac{1}{m_{i}}\left[p_{v i} \omega_{i} \boldsymbol{\varphi}^{\perp \top}\left(\theta_{i}\right) \mathbf{e}_{i}+p_{v i} \varphi^{\top}\left(\theta_{i}\right) \dot{\mathbf{e}}_{i}+d_{v i} \dot{v}_{i}\right],
$$

where we have defined $\varphi^{\perp}$ as the annihilator of $\varphi\left(\theta_{i}\right)$, i.e., $\varphi^{\top}\left(\theta_{i}\right) \varphi^{\perp}\left(\theta_{i}\right)=\varphi^{\perp \top}\left(\theta_{i}\right) \varphi\left(\theta_{i}\right)=0$, and it is given by

$$
\varphi^{\perp}\left(\theta_{i}\right)=\left[\begin{array}{c}
-\sin \left(\theta_{i}\right) \\
\cos \left(\theta_{i}\right)
\end{array}\right] \text {. }
$$

Moreover, since $\dot{\theta}_{i}=\omega_{i}, \dot{\varphi}\left(\theta_{i}\right)=\omega_{i} \varphi^{\perp}\left(\theta_{i}\right)$ and $\dot{\varphi}^{\perp}\left(\theta_{i}\right)=$ $-\omega_{i} \varphi\left(\theta_{i}\right)$.

The fact that $v_{i} \in \mathcal{L}_{\infty} \cap \mathcal{L}_{2},\left\|\overline{\mathbf{z}}_{i}-\overline{\mathbf{z}}_{j}\right\| \in \mathcal{L}_{\infty}$ and (by assumption) $\omega_{i} \in \mathcal{L}_{\infty}$ ensure that $\ddot{v}_{i} \in \mathcal{L}_{\infty}$ and thus $\dot{v}_{i}$ is uniformly continuous. This completes the proof.

Remark 2: A key step in the solution of the (LFP) problem is the design of the function $\alpha_{i}\left(t, \theta_{i}, \mathbf{e}_{i}\right)$. On one hand, from Proposition $1, \alpha_{i}$ has to be a bounded time-varying function to ensure that $\omega_{i} \in \mathcal{L}_{\infty}$. Since $\lim _{t \rightarrow \infty} \varphi^{\top}\left(\theta_{i}(t)\right) \mathbf{e}_{i}(t)=0$ does not imply that $\lim _{t \rightarrow \infty} \mathbf{e}_{i}(t)=0$, on the other hand, $\alpha_{i}$ has to be designed such that if $\alpha_{i}$ and $\boldsymbol{\varphi}^{\top}\left(\theta_{i}\right) \mathbf{e}_{i}$ converge to zero then $\mathbf{e}_{i}$ converges to zero.

In order to accomplish the second objective in Remark 2 , we first note that

$$
\left[\begin{array}{c}
\varphi^{\top}\left(\theta_{i}\right) \\
\varphi^{\perp \top}\left(\theta_{i}\right)
\end{array}\right]=\left[\begin{array}{cc}
\cos \left(\theta_{i}\right) & \sin \left(\theta_{i}\right) \\
-\sin \left(\theta_{i}\right) & \cos \left(\theta_{i}\right)
\end{array}\right]
$$

is a full-rank matrix. Proposition 2 establishes the conditions under which $\lim _{t \rightarrow \infty} \varphi^{\top}\left(\theta_{i}(t)\right) \mathbf{e}_{i}(t)=0$. Hence, if we establish that $\lim _{t \rightarrow \infty} \varphi^{\stackrel{\perp \rightarrow}{\perp \rightarrow \infty}}\left(\theta_{i}(t)\right) \mathbf{e}_{i}(t)=0$ then we will have that

$$
\lim _{t \rightarrow \infty}\left[\begin{array}{c}
\varphi^{\top}\left(\theta_{i}(t)\right) \\
\varphi^{\perp \top}\left(\theta_{i}(t)\right)
\end{array}\right] \mathbf{e}_{i}(t)=\mathbf{0}_{2},
$$

whose only possible solution is $\lim _{t \rightarrow \infty} \mathbf{e}_{i}(t)=\mathbf{0}_{2}$.

After such reasoning, we design of $\alpha_{i}$ as

$$
\alpha_{i}\left(t, \theta_{i}, \mathbf{e}_{i}\right):=k_{\alpha i} f_{i}(t) \boldsymbol{\varphi}^{\perp \top}\left(\theta_{i}\right) \mathbf{e}_{i},
$$

where $k_{\alpha i}>0$ and $f_{i}(t)$ is any time-varying function such that $f_{i} \in \mathcal{C}^{2}, f_{i}, \dot{f}_{i}, \ddot{f}_{i} \in \mathcal{L}_{\infty}, \lim _{t \rightarrow \infty} f_{i}(t) \neq 0$ and $\lim _{t \rightarrow \infty} \dot{f}_{i}(t) \neq 0$.

$\rightarrow$ Remark 3: There exist several functions that satisfy the conditions on $f_{i}(t)$, one trivial example is: $f_{i}(t)=$ 
$A_{i} \sin \left(\omega_{i}^{\star} t\right)$, where $A_{i}, \omega_{i}^{\star} \in \mathbb{R}$ are the magnitude and the frequency, respectively.

We are now ready to establish the main result of this work.

Theorem 1: Controller (3), (6), (8) and (15) solves the (LFP) problem, provided that (11) and (12) hold.

Proof. First we invoke the first part of Proposition 2 to show that $v_{i} \in \mathcal{L}_{\infty} \cap \mathcal{L}_{2},\left\|\overline{\mathbf{z}}_{i}-\overline{\mathbf{z}}_{j}\right\| \in \mathcal{L}_{\infty}$ and $\lim _{t \rightarrow \infty} v_{i}(t)=0$.

These bounded signals imply that $\mathbf{e}_{i} \in \mathcal{L}_{\infty}^{t \rightarrow \infty}$. Hence, the function $\alpha_{i}$ defined in (15) is bounded. Therefore, we may invoke item 2) of Proposition 1 to conclude that $\dot{\omega}_{i}, \omega_{i} \in \mathcal{L}_{\infty}$. Hence, following the second part of Proposition 2, it holds that $\lim _{t \rightarrow \infty} \boldsymbol{\varphi}^{\top}\left(\theta_{i}(t)\right) \mathbf{e}_{i}(t)=0$.

Moreover, in Proposition 2, it has also been proved that $\lim _{t \rightarrow \infty} \dot{v}_{i}(t)=0$. Hence

$$
\lim _{t \rightarrow \infty} \int_{0}^{t} \ddot{v}_{i}(\sigma) d \sigma=\lim _{t \rightarrow \infty} \dot{v}_{i}(t)-\dot{v}_{i}(0)=-\dot{v}_{i}(0) .
$$

So, if we prove that $\ddot{v}_{i}$ is uniformly continuous then

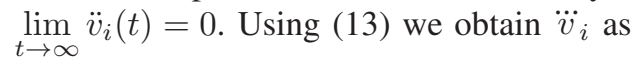

$$
\begin{aligned}
\frac{m_{i}}{p_{v i}} \dddot{v}_{i}= & -\dot{\omega}_{i} \boldsymbol{\varphi}^{\perp \top}\left(\theta_{i}\right) \mathbf{e}_{i}+\omega_{i}^{2} \boldsymbol{\varphi}^{\top}\left(\theta_{i}\right) \mathbf{e}_{i}-2 \omega_{i} \varphi^{\perp \top}\left(\theta_{i}\right) \dot{\mathbf{e}}_{i} \\
& -\boldsymbol{\varphi}^{\top}\left(\theta_{i}\right) \ddot{\mathbf{e}}_{i}-\frac{d_{v i}}{p_{v i}} \ddot{v}_{i} .
\end{aligned}
$$

Assumption $\mathbf{A 2}$ and all the bounded signals imply that $\dddot{v}_{i} \in \mathcal{L}_{\infty}$. Thus, $\lim _{t \rightarrow \infty} \ddot{\ddot{v}}_{i}(t)=0$. Using the previous signal chasing method and invoking systematically Barbalat's Lemma, it can also be concluded that $\lim _{t \rightarrow \infty} \dddot{v}_{i}(t)=0$ and that $\lim _{t \rightarrow \infty} \frac{d}{d t} \dddot{v}_{i}(t)=0$.

Now, $\frac{d}{d t} \dddot{v}_{i}$ is given by

$$
\begin{aligned}
\frac{m_{i}}{p_{v i}} \frac{d}{d t} \dddot{v}_{i}= & -\ddot{\omega}_{i} \varphi^{\perp \top}\left(\theta_{i}\right) \mathbf{e}_{i}-3 \dot{\omega}_{i} \varphi^{\perp \top}\left(\theta_{i}\right) \dot{\mathbf{e}}_{i}+\omega_{i}^{3} \varphi^{\perp \top}\left(\theta_{i}\right) \mathbf{e}_{i} \\
& -3 \omega_{i}\left[\boldsymbol{\varphi}^{\perp \top}\left(\theta_{i}\right) \ddot{\mathbf{e}}_{i}-\dot{\omega}_{i} \boldsymbol{\varphi}^{\top}\left(\theta_{i}\right) \mathbf{e}_{i}-\omega_{i} \boldsymbol{\varphi}^{\top}\left(\theta_{i}\right) \dot{\mathbf{e}}_{i}\right] \\
& -\boldsymbol{\varphi}^{\top}\left(\theta_{i}\right) \dddot{\mathbf{e}}_{i}-\frac{d_{v i}}{p_{v i}} \dddot{v}_{i} .
\end{aligned}
$$

Since $v_{i}, \dot{v}_{i}, \ddot{v}_{i}, \dddot{v}_{i}, \frac{d}{d t} \dddot{v}_{i}$ and $\varphi^{\top}\left(\theta_{i}\right) \mathbf{e}_{i}$ converge to zero, they imply that the terms $\omega_{i} \varphi^{\perp \top}\left(\theta_{i}\right) \mathbf{e}_{i}, \dot{\omega}_{i} \varphi^{\perp \top}\left(\theta_{i}\right) \mathbf{e}_{i}$ and $\ddot{\omega}_{i} \varphi^{\perp \top}\left(\theta_{i}\right) \mathbf{e}_{i}$ also converge to zero. Substituting $\ddot{\omega}_{i}$, given by

$$
\frac{I_{i}}{k_{\alpha i}} \ddot{\omega}_{i}=-\frac{p_{\omega i}}{k_{\alpha i}} \dot{e}_{\theta i}-\frac{d_{\omega i}}{k_{\alpha i}} \dot{\omega}_{i}-f_{i} \omega_{i} \varphi^{\top} \mathbf{e}_{i}+f_{i} \varphi^{\perp \top} \dot{\mathbf{e}}_{i}+\dot{f}_{i} \varphi^{\perp \top} \mathbf{e}_{i},
$$

in the first right hand side term of (17), i.e., $\ddot{\omega}_{i} \varphi^{\perp \top}\left(\theta_{i}\right) \mathbf{e}_{i}$, supports the fact that the resulting term $\dot{f}_{i}\left(\varphi^{\perp \top} \mathbf{e}_{i}\right)^{2}$ also converges to zero. The design of $f_{i}(t)$ guarantees that $\dot{f}_{i}(t)$ does not vanish. Hence $\lim _{t \rightarrow \infty} \varphi^{\perp \top}\left(\theta_{i}(t)\right) \mathbf{e}_{i}(t)=0$, as required. This establishes the proof that $\lim _{t \rightarrow \infty} \mathbf{e}_{i}(t)=0$ and this, in turn, implies that $\lim _{t \rightarrow \infty} \overline{\mathbf{z}}_{i}(t)=\overline{\mathbf{z}}_{c}$. The proof is completed by invoking item 3 ) of Proposition 1 .

\section{Simulations}

In order to show the effectiveness of the proposed scheme, this section presents simulation results with six differential

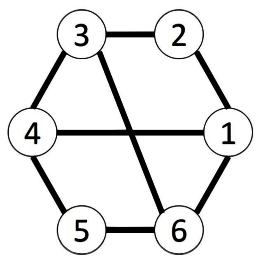

Fig. 2. Communication topology and formation pattern.

drive robots, whose communication topology and formation pattern are depicted in Fig. 2. For simplicity, the physical parameters are the same for all the robots, the mass is $m_{i}=$ $10 \mathrm{~kg}$, the moment of inertia is $I_{i}=3 \mathrm{Kgm}^{2}$, the distance between point $\mathbf{Q}$ and the wheels is $R=0.3 \mathrm{~m}$ and the radius of the wheels is $r=0.05 \mathrm{~m}$. These values are the same as those used in [23].

TABLE I

INITIAL POSITIONS FOR THE ROBOTS AND DESIRED RELATIVE POSITION.

\begin{tabular}{c|cccccc} 
& 1 & 2 & 3 & 4 & 5 & 6 \\
\hline$x_{i}(0)$ & 5 & 7 & 7 & 3 & 1 & 1 \\
$y_{i}(0)$ & 2 & 5.5 & 3.5 & 2 & 3.5 & 5.5 \\
$\theta_{i}(0)$ & 0 & $-\frac{\pi}{4}$ & $-\frac{\pi}{2}$ & $\frac{\pi}{4}$ & $\frac{\pi}{2}$ & $\frac{\pi}{4}$ \\
\hline$\delta_{x i}$ & 2 & 1 & -1 & -2 & -1 & 1 \\
$\delta_{y i}$ & 0 & 2 & 2 & 0 & -2 & -2
\end{tabular}

The initial velocities are all set to zero and the initial positions, together with the relative desired positions, are shown in Table I. The controller gains have been set to $p_{v i}=300, d_{v i}=600, p_{\omega i}=30, d_{\omega i}=60, k_{\alpha i}=150$ these gains satisfy the bounds (11) and (12) with an upperbound ${ }^{*} T_{j i}=0.33$. The transmission delays, depicted in Fig. 3, follow a normal Gaussian distribution with a mean of 0.2 and a variance of 0.001 . The time-varying function $f_{i}(t)$ is the same for all robots and it is given by $f_{i}(t)=$ $\frac{5}{2}+2 \pi \sin (t)+\pi \sin (2 t)+\frac{2}{3} \sin (3 t)$.

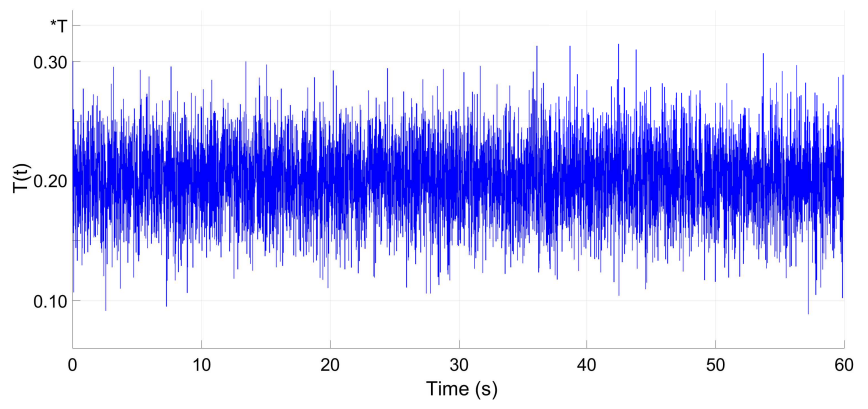

Fig. 3. Variable time-delay with an upper-bound at ${ }^{*} T$.

The position trajectories of the non-holonomic robots are shown in Fig. 4, from which one can see that the desired hexagon formation is achieved. The orientation behavior is presented in Fig. 5, it takes about 30 seconds for the network of robots to reach a consensus. Hence, the (LFP) is solved, as desired. 


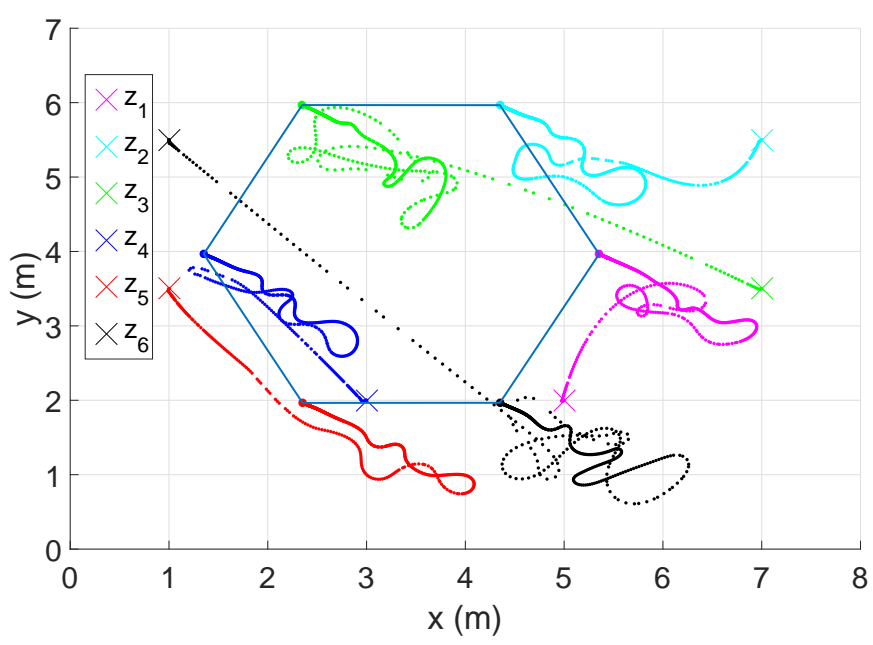

Fig. 4. Position trajectories in the Cartesian $x y$-plane.

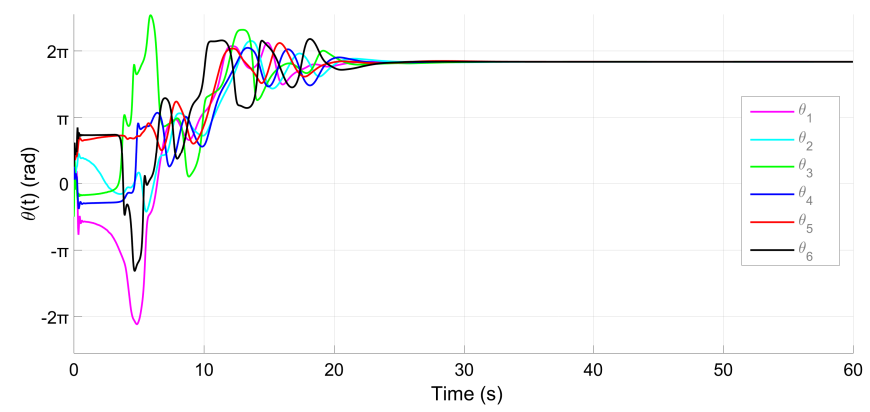

Fig. 5. Robot orientations.

\section{CONCLUSIONS}

In this work we solve the leaderless consensus-based formation control of swarms of a class of nonholonomic mobile robots. We consider the real scenario where the robots are modeled by second order dynamics and where unreliable communications that exhibit variable time-delays arise. The proposed smooth time-varying controller is a simple to implement Proportional plus damping $(\mathrm{P}+\mathrm{d})$ scheme.

Future work include the solution to the leader-follower formation control and the design of a controller that does not rely on velocity measurements. In order for the robots not to collide while they find their respective position in the formatin, we also plan to include a collision avoidance term in the proposed controller.

\section{ACKNOWLEDGMENT}

This article is supported by the Mexican CONACyT Basic Scientific Research grant CB-282807; by the MexicanFrench ECOS-NORD project 249543; by the University of Paris Saclay; and by the Government of Russian Federation grant 074-U01.

\section{REFERENCES}

[1] A. Jadbabaie, J. Lin, and A. Morse, "Coordination of groups of mobile autonomous agents using nearest neighbor rules," IEEE Transactions on Automatic Control, vol. 48, no. 6, pp. 988-1001, 2003.
[2] R. Olfati-Saber and R. Murray, "Consensus problems in networks of agents with switching topology and time-delays," IEEE Transactions on Automatic Control, vol. 49, no. 9, pp. 1520-1533, 2004.

[3] E. Nuño, R. Ortega, L. Basañez, and D. Hill, "Synchronization of networks of nonidentical Euler-Lagrange systems with uncertain parameters and communication delays," IEEE Transactions on Automatic Control, vol. 56, no. 4, pp. 935-941, 2011.

[4] E. Nuño and R. Ortega, "Achieving consensus of Euler-Lagrange agents with interconnecting delays and without velocity measurements via passivity-based control," IEEE Transactions on Control Systems Technology, vol. 26, no. 1, pp. 222-232, 2018.

[5] Y. Cao and W. Ren, Distributed Coordination of Multi-agent Networks: Emergent Problems, Models, and Issues. Springer-Verlag, 2011.

[6] H. Wang, "Consensus of networked mechanical systems with communication delays: A unified framework," IEEE Transactions on Automatic Control, vol. 59, no. 6, pp. 1571-1576, 2014.

[7] T. Hatanaka, N. Chopra, M. Fujita, and M. Spong, Passivity-Based Control and Estimation in Networked Robotics, ser. Communications and Control Engineering. Springer, 2015.

[8] D. V. Dimarogonas and K. J. Kyriakopoulos, "On the rendezvous problem for multiple nonholonomic agents," Automatic Control, IEEE Transactions on, vol. 52, no. 5, pp. 916-922, 2007.

[9] Z. Lin, B. Francis, and M. Maggiore, "Necessary and sufficient graphical conditions for formation control of unicycles," Automatic Control, IEEE Transactions on, vol. 50, no. 1, pp. 121-127, 2005.

[10] C. Yang, W. Xie, C. Lei, and B. Ma, "Smooth time-varying formation control of multiple nonholonomic agents," in Proceedings of the 2015 Chinese Intelligent Systems Conference. Springer, 2016, pp. 283-291.

[11] A. Ajorlou, M. M. Asadi, A. G. Aghdam, and S. Blouin, "Distributed consensus control of unicycle agents in the presence of external disturbances," Systems \& Control Letters, vol. 82, pp. 86-90, 2015.

[12] Z. Peng, G. Wen, A. Rahmani, and Y. Yu, "Distributed consensusbased formation control for multiple nonholonomic mobile robots with a specified reference trajectory," International Journal of Systems Science, vol. 46, no. 8, pp. 1447-1457, 2015.

[13] A. Bautista-Castillo, C. Lopez-Franco, and E. Nuno, "Consensusbased formation control for multiple nonholonomic robots," in Accepted in 2016 IEEE International Autumn Meeting on Power, Electronics and Computing (ROPEC). IEEE, 2016.

[14] J. Jin and N. Gans, "Collision-free formation and heading consensus of nonholonomic robots as a pose regulation problem," Robotics and Autonomous Systems, vol. 95, no. 9, pp. 25-36, 2017.

[15] M. Maghenem, A. loría, and E. Panteley, "A robust $\delta$-persistently exciting controller for leader-follower tracking-agreement of multiple vehicles," European Journal of Control, vol. 40, no. 3, pp. 1-12, 2018.

[16] M. Maghenem, A. Bautista-Castillo, E. Nuño, A. Loría, and E. Panteley, "Consensus of multi-agent systems with nonholonomic restrictions via Lyapunov's direct method." IEEE Control Systems Letters, vol. 3, no. 2, pp. 344-349, 2019.

[17] M. Maghenem, A. Bautista-Castillo, E. Nuño, A. loría, and E. Panteley, "Consensus-based formation control of nonholonomic robots using a strict Lyapunov function," IFAC-PapersOnLine, vol. 50, no. 1, pp. 2439-2444, 2017, IFAC World Congress, Toulouse, France.

[18] W. Dong and J. A. Farrell, "Consensus of multiple nonholonomic systems," in Decision and Control, 2008. CDC 2008. 47th IEEE Conference on. IEEE, 2008, pp. 2270-2275.

[19] E. Nuño, I. Sarras, and L. Basañez, "Consensus in networks of nonidentical Euler-Lagrange systems using $\mathrm{P}+\mathrm{d}$ controllers," IEEE Transactions on Robotics, vol. 26, no. 6, pp. 1503-1508, 2013.

[20] C. I. Aldana, E. Romero, E. Nuño, and L. Basañez, "Pose consensus in networks of heterogeneous robots with variable time delays," International Journal of Robust and Nonlinear Control, vol. 25, no. 14, pp. 2279-2298, 2015.

[21] E. Nuño, "Consensus of Euler-Lagrange systems using only position measurements," IEEE Transactions on Control of Network Systems., vol. 5, no. 1, pp. 489-498, 2018.

[22] H. Wang, "Integral-cascade framework for consensus of networked Lagrangian systems," 2018. [Online]. Available: https://www.researchgate.net/publication/325997304Integral-CascadeFramework-for-Consensus-of-Networked-Lagrangian-Systems.pdf

[23] K. Shojaei, A. M. Shahri, A. Tarakameh, and B. Tabibian, "Adaptive trajectory tracking control of a differential drive wheeled mobile robot," Robotica, vol. 29, no. 3, pp. 391-402, 2011. 